\title{
Automatic Detection of the Number of Raypaths
}

\author{
Longyu Jiang \\ GIPSA-Lab / DIS \\ Grenoble INP, France \\ Email: Long-Yu.Jiang@gipsa-lab.grenoble-inp.fr
}

\author{
Jérôme I. Mars \\ GIPSA-Lab / DIS \\ Grenoble INP, France
}

\begin{abstract}
In this paper, an Exponential Fitting Test (EFT) is presented in the context of ocean acoustic tomography for detecting the number of the raypaths. It is based on the fact that the profile of the ordered eigenvalues fits an exponential law for white Gaussian noise and small samples. The number of raypaths could be detected when a mismatch occurs between the observed profile and the exponential model. Its performance is studied with simulated experiment datas. EFT works for the case of small number of samples when information theoretic criterias fail.
\end{abstract}

\section{INTRODUCTION}

In the ocean, acoustic tomography is used to image the sound speed variations. A common way to proceed is to take advantage of the multi-path properties of the wavefield [1]. Each path provides information on the variation of sound speed distribution. However, multi-path propagation produces interferences between raypaths. In order to separate the different paths and extract more observations, high resolution methods are applied for ocean acoustic tomography [2]. The same as general situation for array processing, if the model order is poorly selected, the separation may be hampered. Two commonly suggested approaches for this task are the Akaike information criterion (AIC) and the minimum description length (MDL) [3]. With ideal assumptions, the MDL criterion is shown to be asymptotically consistent, whereas the AIC tends to overestimate the order of model. However, these ideal assumptions may not be fullfilled in practice and several factors could result in the smallest eigenvalues being dispersed (for example: reduction number of samples or low SNR). Both the AIC and the MDL tend to overestimate or underestimate the order of model. For analysing the performance and evaluating the probability of over or under estimation of AIC and MDL, several theoretical results are presented [4] [5].

Recently, a criteria based on eigenvalue ratios is used to look for an eigenvalue gap between the noise and the signal eigenvalues [5]. As a followed method, a method exploiting the exponetial profile of the orderd noise eigenvalue, which is first introduced in [6], is applied to obtain an estimation of the number of significant targets in time reversal imaging [7].

Since a long duration of the received signal in the context of ocean acoustic tomography is unavailable, an EFT using short samples is proposed to determine model order of high resolution method in the following part, which would be considered robust and applicable. In this paper, short samples means that the number of samples equals the number of sensors.

\section{Exponential Fitting Test}

\section{A. Signal model}

The model is built on an acoustic field composed of $p$ raypaths on a vertical antenna of $M$ sensors $(p<M)$. The temporal signal received on sensors is modelized as:

$$
\mathbf{x}(t)=\mathbf{A e}(t)+\mathbf{n}(t)
$$

With:

- $\mathbf{x}(t)$ : a $M \times 1$ observation vector.

- $\mathbf{A}$ : the matrix of the $p$ steering vectors.

- $\mathbf{e}(t)$ : the raypaths vector of size $p \times 1$.

- $\mathbf{n}(t)$ : additive white Gaussian noise of size $M \times 1$ with distribution $N\left(0, \sigma^{2} \mathbf{I}\right)$, which is assumed to be uncorrelated with the signals.

The observation covariance matrix $\mathbf{R}_{x}$ can be written as:

$$
\mathbf{R}_{x}=E\left[\mathbf{x}(t) \mathbf{x}^{*}(t)\right]=\mathbf{R}_{y}+\mathbf{R}_{n}=\mathbf{A} \mathbf{R}_{s} \mathbf{A}^{*}+\sigma^{2} \mathbf{I}
$$

\section{B. Eigenvalues Profile Under Noise Only Assumption}

In order to establish the mean profile of the decreasing noise eigenvalues, we need to calculate the expectation of each eigenvalue. For the zero-mean white Gaussian noise with power $\sigma^{2}$, the sample covariance matrix has a Wishart distribution with $N$ degrees of freedom. It is a multivariate generalization of $\chi^{2}$ distribution and depends on $M, N, \sigma^{2}$. In this case, the joint probability of an ordered eigenvalues and the distribution of each eigenvalue can be obtained [8] [9], but this requires zonal polynomials and give untractable results. Thus, we use an alternative approach to approximate the mean profile of ordered noise eigenvalue with the help of first and second order moments of the eigenvalues.

The simulation result ( Fig.1.) turns out that an exponential law could be a good approximation to the mean profile of eigenvalues:

$$
\lambda_{i}=\lambda_{1} r_{M, N}^{i-1}
$$

The error of the covariance matrix $\varphi$,

$$
\varphi=\hat{\mathbf{R}}_{x}-\mathbf{R}_{x}=\hat{\mathbf{R}}_{x}-\sigma^{2} \mathbf{I}
$$

The noise eigenvalue profile can be found by considering the first and second moments of $\operatorname{tr}[\varphi]$.

Because of $E(\operatorname{tr}[\varphi])=0$, it could be obtained:

$$
M \sigma^{2}=\sum_{i=1}^{M} \lambda_{i}
$$


and $E\left(\operatorname{tr}\left[\hat{\mathbf{R}}_{x}-\mathbf{R}_{x}\right]^{2}\right)=E\left(\operatorname{tr}\left[\varphi_{i j}\right]^{2}\right)=M^{2} \frac{\sigma^{4}}{N}$, it follows that:

$$
M^{2} \frac{\sigma^{4}}{N}=\sum_{i=1}^{M}\left(\lambda_{i}-\sigma^{2}\right)^{2}
$$

From combination of Eqs. (3), (5) and (6), we obtain :

$$
\lambda_{1}=M J_{M} \sigma^{2}
$$

where:

$$
J_{M}=\frac{1-r}{1-r^{M}}
$$

and the decay rate is obtained from the following equation:

$$
\frac{M+N}{M N}=\frac{(1-r)\left(1+r^{M}\right)}{\left(1-r^{M}\right)(1+r)}
$$

By substituing $r=e^{-2 a}$, Eq. (9) becomes:

$$
\frac{\operatorname{Mtanh}(a)-\tanh (a)}{\operatorname{Mtanh}(a)}=\frac{1}{N}
$$

where tanh is the hyperbolic tangent function.

By using an order 4 expansion of Eq. (10), the following biquadratic equation is produced as:

$$
\sigma^{4}-\frac{15}{M^{2}+2} a^{2}+\frac{45 M^{2}}{N\left(M^{2}+1\right)\left(M^{2}+2\right)}=0
$$

The positive solution of Eq. (14) is given by:

$$
a(M, N)=\sqrt{\frac{15}{2\left(M^{2}+2\right)}\left[1-\sqrt{\frac{4 M\left(M^{2}+2\right)}{5 N\left(M^{2}-1\right)}}\right]}
$$

\section{Principle of the Recursive Exponential Fitting Test}

With the assumption of $p$ decorrelated or partly correlated raypaths, the recursive exponential fitting test is mainly based on the comparation between the ordered profile of the normalized eigenvalues of the sample covariance matrix of observation and the theoretical profile of the ordered noise eigenvalues. A break point occurs when signal eigenvalue appears.

The recursive test is started from $P=1$. Assuming the $P$ smallest eigenvalues are noise eigenvalues, the previous eigenvalue $\lambda_{M-P}$ is tested to see that it corresponds to noise or a raypath. For each value of $P$, the test is done by two steps:

Step 1: predict the value of $\lambda_{M-P}$ according to exponential model (Eq. 7):

$$
\hat{\lambda}_{M-P}=(P+1) J_{P+1} \hat{\sigma}^{2}
$$

where:

$$
J_{P+1}=\frac{1-r_{P+1}}{1-r_{P+1}^{P+1}}
$$

$$
\hat{\sigma}^{2}=\frac{1}{P+1} \sum_{i=0}^{P} \lambda_{i}
$$

The prediction equation is obtained by the combination of Eq. (14), Eq. (15) and Eq. (13):

$$
\hat{\lambda}_{M-P}=J_{P+1} \sum_{i=0}^{P} \lambda_{M-i}
$$

$r_{P+1}$ is calculated by first getting $a$ using Eq. (12) and $r=e^{-2 a}$, where $(P+1)$ should be instead of $M$.

Step 2: In this step, define the two hypotesis as follows:

- $H_{P+1}: \lambda_{M-P}$ is a noise eigenvalue.

- $\bar{H}_{P+1}: \lambda_{M-P}$ is a raypath eigenvalue.

In order to decide between these hypotesis, the absolute error of $\lambda_{M-P}$ and $\hat{\lambda}_{M-P}$ is calculated and then be compared with a threshold $\eta_{P}$. That is:

$$
\begin{aligned}
& H_{P+1}:\left|\lambda_{M-P}-\hat{\lambda}_{M-P}\right| \leq \eta_{P} \\
& H_{P+1}:\left|\lambda_{M-P}-\hat{\lambda}_{M-P}\right|>\eta_{P}
\end{aligned}
$$

The empirical distribution of the noise-only eigenvalue profile can be used to find a suitable threshold. We generate 10000 realizations for an array of $M=15$ sensors and $N=15$ samples. The mean profile of noise eigenvalue can be computed. (It is represented by the middle cuve in Fig. 1. ) For each eigen index, there exists upper and lower noise eigenvalues. Half of the distance between them for eigen index $P$ could be taken as the threshold $\eta_{P}$.

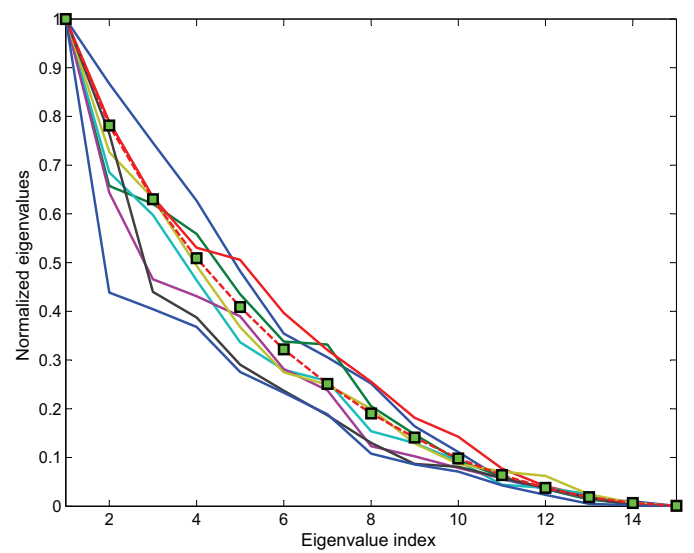

Fig. 1. Profile of orderd noise eigenvalues for eight realizations

\section{SimULATIONS}

We illustrate the performace of EFT method with simulation datas. For various RSB and different number of samples, eight experiments are realised. In these experiments, five coherent rays arrive on 15 sensors. Because of the function of noise, these rays could be taken as partly correlated rays. Fig. 2., Fig. 3. and Fig. 4. . respectively show the received rays and ordered 
eigenvalue profile for a certain group of RSB and samples. As comparative methods, AIC and MDL are applied to these experiments. Considering asymptotically consistent property of AIC and MDL, we choose $N=1025$, which is much larger that the number of sensors. In this case, AIC and MDL still overestimate or underestimate the number of rayspath. The possibility of overestimation or underestimation mainly depends on the distribution of dispered eigenvalue. It obeys the qualitative resluts in [5]. For fixed number of samples, overmodeling becomes more likely for increasing the noise eigenvalue dispersion. For fixed dispersion, overmodeling becomes more likely for increasing the number of data samples. Undermodeling may happen in the cases where the signal eigenvalues are not well separated from the noise eigenvalues and the noise eigenvalues are clustered sufficiently closely. The results are shown by Table 1 . The detection results for the case of $N=15$ by EFT turns out that EFT could detect relatively correctly for decorrelated or partly correlated rays. For the case of coherent rays ( the example of $\mathrm{SNR}=10 \mathrm{~dB}$ ), because of the rank deficiency of covariance matrix, EFT tends to underestimate. This will be study in our futher work. AIC and MDL don't work for so short samples.

\begin{tabular}{|c|c|c|c|c|}
\cline { 2 - 5 } \multicolumn{1}{c|}{} & SNR (dB) & AIC & MDL & EFT \\
\hline \multirow{3}{*}{$N=1025$} & 10 & 14 & 14 & - \\
\cline { 2 - 5 } & 5 & 14 & 12 & - \\
\cline { 2 - 5 } & -5 & 7 & 1 & - \\
\cline { 2 - 5 } & -10 & 1 & 0 & - \\
\hline \multirow{3}{*}{$N=15$} & 10 & - & - & 3 \\
\cline { 2 - 5 } & 5 & - & - & 4 \\
\cline { 2 - 5 } & -5 & - & - & 6 \\
\cline { 2 - 5 } & -10 & - & - & 5 \\
\hline
\end{tabular}

TABLE I

NUMBER OF RAYPATHS DETECTED BY AIC, MDL AND EFT (THE REAL NUMBER OF RAYPATHS $p=5$ )

\section{CONCLUSION}

We propose an Exponential Fitting Test to detect the number of rayspaths in ocean acoustic tomography. It uses the property of the ordered eigenvalue profile, which could be approximated to an exponential law in the case of noise-only, to detect a breakpoint between ordered noise eigenvalue profile and ordered eigenvalue profile of observation. It enables to give a relative correct detection for short samples while AIC and MDL fail. Next, it will be applied to real data and combined with high resolution processing [2] to extract raypaths for ocean acoustic tomography.

\section{REFERENCES}

[1] W. Munk, P. Worcester, and C. Wunsch. Ocean Acoustic Tomography. Cambridge monographs on mechanics, Cambridge, 1995.

[2] L. Jiang, F. Aulanier, G. Touzé, B. Nicolas and J. I. Mars. Raypath Separation with High Resolution Processing. in Proceeding of Oceans 11 IEEE Santander, Spain, Jun. 6-9, 2011.

[3] M. Wax and T. Kailath. Detection of signals by information theoretic criteria. IEEE Trans. Acoust. Speech Signal Processing, 33(2): 387-392, 1985.

[4] W. Xu and M. Kaveh. Analysis of the performance and sensitivity of eigendecomposition-based detectors. IEEE Trans. Signal Processing, 43: 1413-1426, 1995

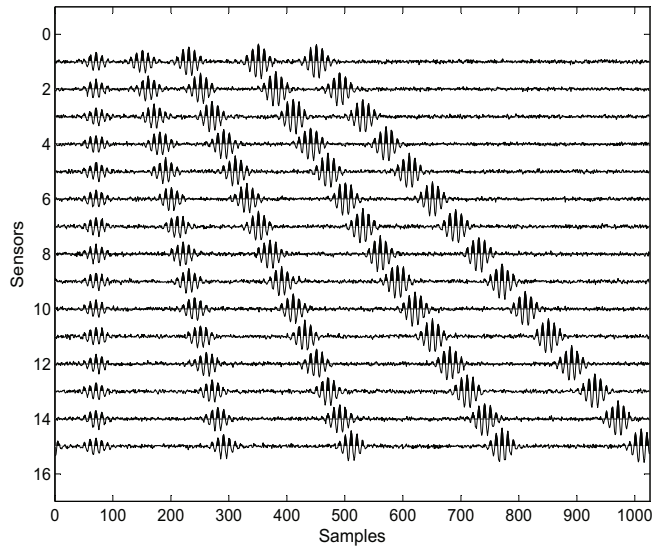

(a) signals

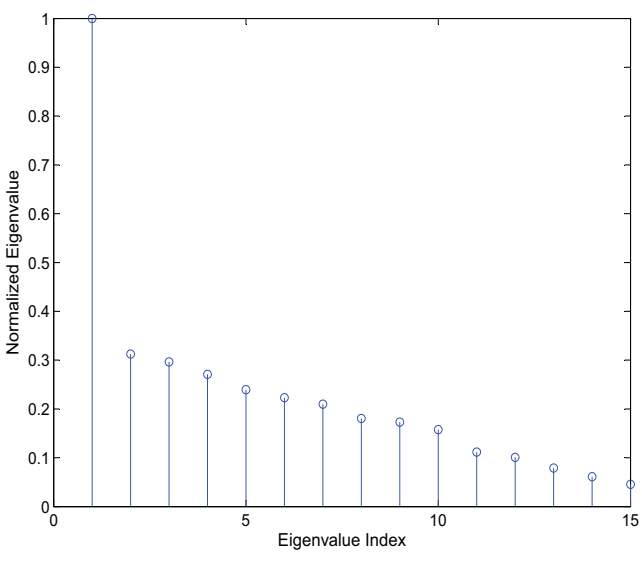

(b) $N=1025$

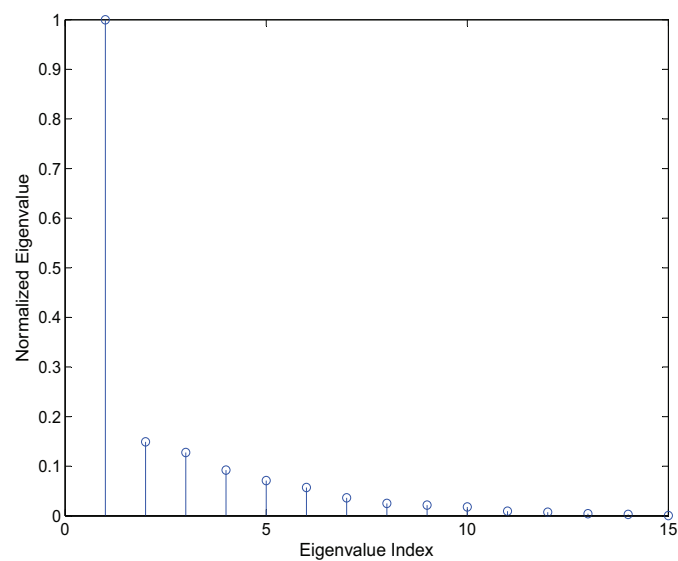

(c) $N=15$

Fig. 2. Profile of orderd eigenvalues $(\mathrm{SNR}=10 \mathrm{~dB})$ 


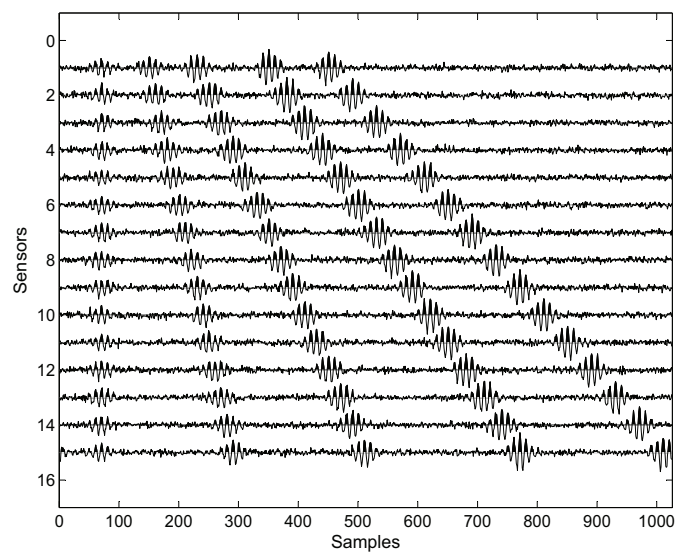

(a) signals

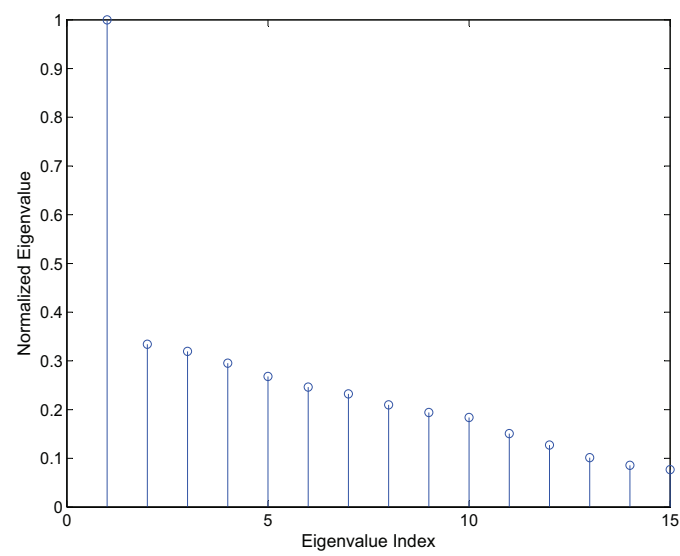

(b) $N=1025$

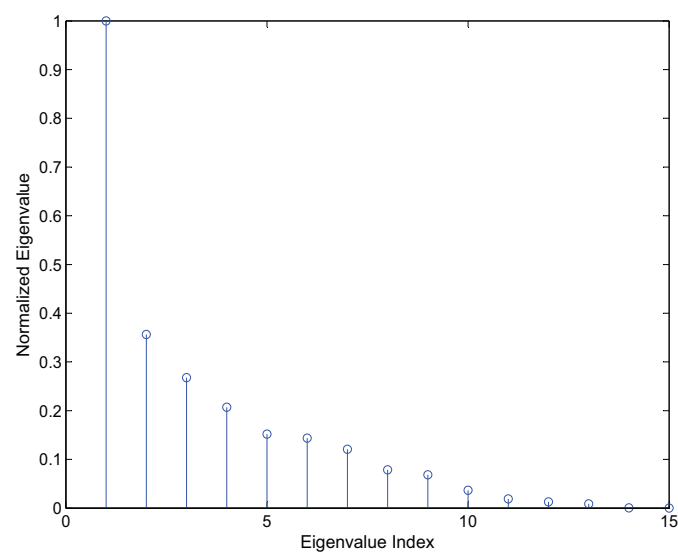

(c) $N=15$

Fig. 3. Profile of orderd eigenvalues $(\mathrm{SNR}=5 \mathrm{~dB})$

[5] A. P. Liavas and P. A. Regalia. On the behavior of information theoretic criteria for model order selection. IEEE Transactions on Signal Processing, 49(8) : 1689-1695, 2001.

[6] J. Grouffaud, P. Larzabal, and H. Clergeot. Some properties of ordered eigenvalues of aWishart matrix: application in detection test and model order selection. ICASSP 5: 2463-2466, 1996.

[7] A. Quinlan, J.-P. Barbot, and P. Larzabal. Automatic determination of the number of targets present when using the time reversal operator. The Journal of the Acoustical Society of America 119(4): 2220-2225, 2006.

[8] N. L. Johnson and S. Kotz. Distributions in Statistics: Continuous Multivariate Distributions. chapter 38-39, John Wiley Sons, New York, NY, USA1972.

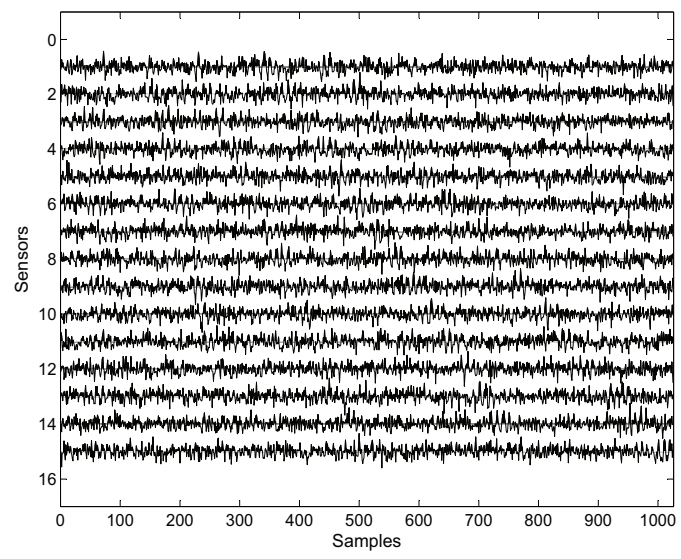

(a) signals

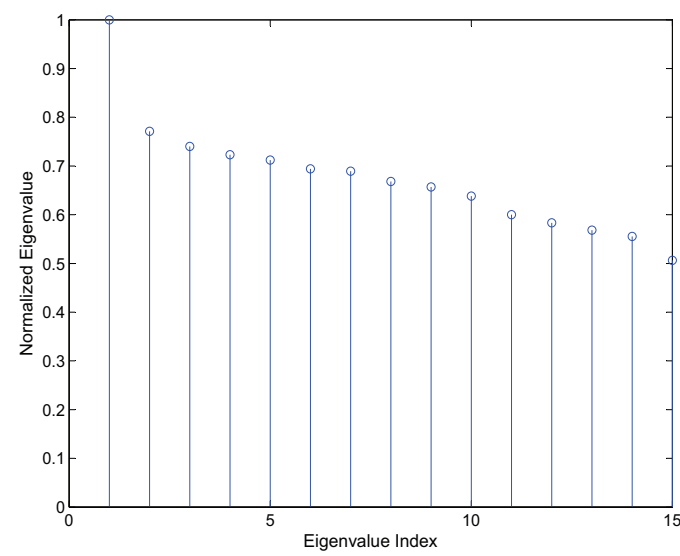

(b) $N=1025$

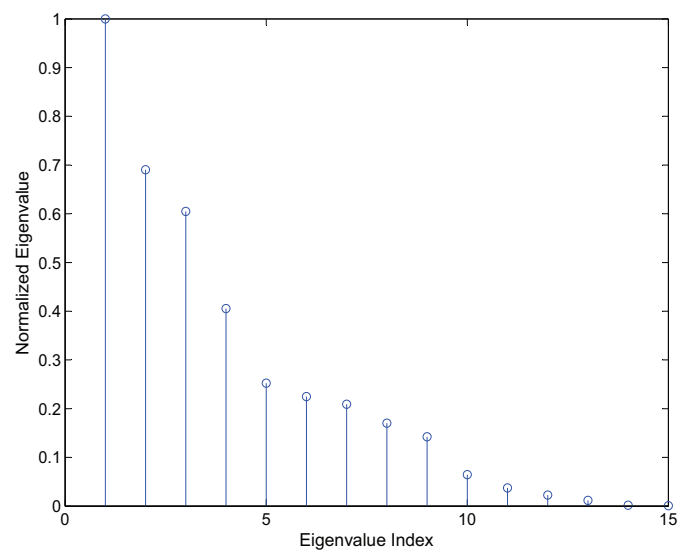

(c) $N=15$

Fig. 4. Profile of orderd eigenvalues $(S N R=-10 \mathrm{~dB})$

[9] P. R. Krishnaiah and F. J. Schurmann. On the evaluation of some distribution that arise in simultaneous tests of the equality of the latents roots of the covariance matrix. Journal of Multivariate Analysis 4, 265282, 1974. 